\title{
Kinetics of Quasiballistic Transport in Nanoscale Semiconductor Structures: Is the Ballistic Limit Attainable at Room Temperature?
}

\author{
Nobuyuki Sano* \\ Institute of Applied Physics, University of Tsukuba, 1-1-1 Tennoudai, Tsukuba, Ibaraki, 305-8573 Japan
}

(Received 2 August 2004; published 6 December 2004)

\begin{abstract}
Electron transport in nanoscale semiconductor structures is theoretically investigated to answer the question of whether or not the ballistic limit is really attainable under room temperature operation. The semiclassical Boltzmann transport equation is solved analytically under the relaxation time approximation for $n^{+}-n-n^{+}$test structures. We demonstrate that the solution of the Boltzmann transport equation exhibits a boundary layer structure near the potential barrier and thus the scatterings in the active region cannot be neglected even in nanoscale structures, as far as they are operated at room temperature under high applied voltages.
\end{abstract}

DOI: $10.1103 /$ PhysRevLett.93.246803

PACS numbers: 73.23.Ad, 72.20.Dp

The channel length in the state-of-the-art metal-oxide-semiconductor field-effect transistors (MOSFETs) now reaches the order of tens of nanometers, which is nearly comparable to or even smaller than a typical mean-free path at room temperature. Electron transport in such small transit regions is expected to be quasiballistic. Ballistic transport, in which the channel electrons are assumed to cross the active region without any scattering, is the extreme case of such nonstationary transport $[1,2]$. In ideal ballistic transport, the shape of the electron distribution function in the channel is given by the combination of the quasiequilibrium Maxwell distributions at the reservoirs (source and drain) and its transport properties have been studied extensively [1-4]. In reality, however, the scattering rate is large (or at least finite) at room temperature in many cases and it is not certain whether or not such a ballistic limit could be naively taken for the kinetic equation such as the Boltzmann transport equation (BTE). In fact, energy dissipation ought to be inevitable in analyzing irreversible transport characteristics [5].

We have recently examined the velocity distribution functions in the channel region of nanoscale semiconductor structures by numerically solving the BTE [6]. It has been shown explicitly that the velocity distribution functions are greatly broadened due to scatterings and rather different from those expected from the ballistic theory. In the present Letter, this discrepancy is theoretically resolved. The BTE is solved analytically under the relaxation time approximation (RTA) and it is shown that the ballistic limit cannot be naively taken because of the mathematical structure of the BTE.

We begin by reviewing the solution of the onedimensional BTE under the RTA. Under steady state, the BTE takes the form

$$
v \frac{\partial f(x, v)}{\partial x}+\frac{e}{m} \frac{d V(x)}{d x} \frac{\partial f(x, v)}{\partial v}=-\frac{f(x, v)-f_{\mathrm{eq}}(x, v)}{\tau(x)},
$$

where $f(x, v)$ is the electron distribution function, $m$ is the electron effective mass, $V(x)$ is the electrostatic potential, $e(>0)$ is the magnitude of the electronic charge, and $\tau(x)$ is the relaxation time. $f_{\text {eq }}(x, v)$ is the local equilibrium distribution function and given by [7] $f_{\mathrm{eq}}(x, v)=$ $n(x) \sqrt{\frac{m}{2 \pi k_{B} T_{0}}} e^{-\left(m v^{2} / 2 k_{B} T_{0}\right)}$, where $k_{B}$ is the Boltzmann constant, $T_{0}$ is the lattice temperature, and $n(x)=$ $\int d v f(x, v)$ is the electron density.

The ballistic solution is usually obtained from Eq. (1) by taking the limit of $\tau(x) \rightarrow \infty$. Since electrons in a lightly doped active region are assumed to be scattering-free, $f(x, v)$ in the ballistic limit is then given by the sum of the quasiequilibrium distribution functions at the reservoirs. Under a typical energy configuration encountered in diodes or field-effect transistors (FETs), as depicted in Fig. $1, f\left(x_{b}, v\right)$ at the potential barrier $(x=$ $\left.x_{b}\right)$ takes the familiar form as $f_{\mathrm{eq}}\left(x_{S}, \sqrt{v^{2}+\frac{2 e \Delta \Phi_{b}}{m}}\right)$ for $v>0$ and $f_{\text {eq }}\left[x_{D},-\sqrt{v^{2}+\frac{2 e\left(\Delta \Phi_{b}+V_{a}\right)}{m}}\right]$ for $v<0$, where $x_{S}$ and $x_{D}$ are the edge of the highly doped left ( $S$ region) and right ( $D$ region) reservoirs, respectively. $V_{a}$ is the applied voltage and $\Delta \Phi_{b}$ is the potential barrier for electrons. The lightly doped $n$ region $\left(x \in\left[x_{S}, x_{D}\right]\right)$ is denoted hereafter as channel. Ballistic transport is, therefore, described as a simple combination of the right- and

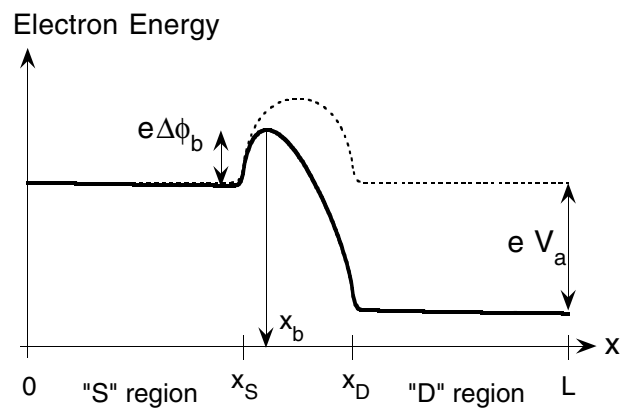

FIG. 1. Typical energy diagram for electrons in conventional devices under the applied voltage $V_{a}$. 
left-moving electrons. We show in Fig. 2 the velocity distribution functions at the top of the electronic potential barrier $\left(x=x_{b}\right)$ obtained by solving numerically the BTE for $n^{+}-n-n^{+}$diodes with various channel lengths $\left(L_{c}=50,30,15 \mathrm{~nm}\right)$ [6]. Notice that the distribution function under the ballistic limit is given by the hemiMaxwellian distribution, whereas the velocity distribution function obtained from the BTE always exhibits non-negligible electrons with negative velocity. Since the number of electrons coming ballistically from the $D$ region is negligible, the electrons with negativecomponent velocity must result from scatterings in the channel region.

The discrepancies in the velocity distribution functions, as observed in Fig. 2, are the manifestation of a serious problem inherent in the ballistic theory. Changing the variables from $(x, v)$ to $(x, \varepsilon)$ with $\varepsilon=m v^{2} / 2-$ $e V(x)(\varepsilon \geq 0)$, Eq. (1) is expressed as

$$
v(x ; \varepsilon) \frac{d f(x ; \varepsilon)}{d x}=-\frac{f(x ; \varepsilon)-f_{\mathrm{eq}}(x ; \varepsilon)}{\tau(x)} .
$$

Since Eq. (2) is linear in $f(x ; \varepsilon)$, the exact solution for the BTE is obtained as

$$
\begin{aligned}
f(x ; \varepsilon)= & e^{-\int_{x_{0}}^{x} d x^{\prime} / v\left(x^{\prime} ; \varepsilon\right) \tau\left(x^{\prime}\right)}\left[f\left(x_{0} ; \varepsilon\right)\right. \\
& \left.+\int_{x_{0}}^{x} d x^{\prime \prime}\left\{\frac{f_{\mathrm{eq}}\left(x^{\prime \prime} ; \varepsilon\right)}{v\left(x^{\prime \prime} ; \varepsilon\right) \tau\left(x^{\prime \prime}\right)} e^{\int_{x_{0}}^{x^{\prime \prime}} d x^{\prime \prime \prime} / v\left(x^{\prime \prime \prime} ; \varepsilon\right) \tau\left(x^{\prime \prime \prime}\right)}\right\}\right] .
\end{aligned}
$$

Notice that electron velocity $v(x ; \varepsilon)$ could be indefinitely small depending on electron's energy $\varepsilon$, whereas the relaxation time $\tau(x)$ is finite at room temperature no matter how small the device size is. Therefore, Eq. (3) is

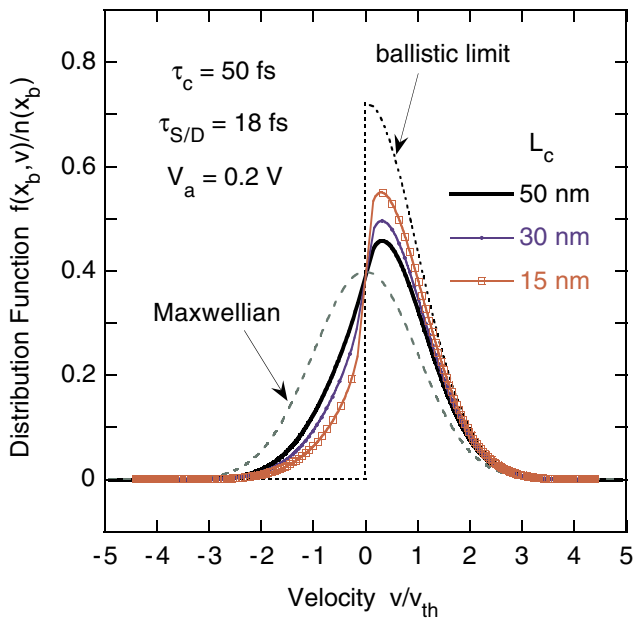

FIG. 2 (color online). Velocity distribution functions at the top of the potential barrier for $n^{+}-n-n^{+}$diodes. The dashed and dotted curves represent the local equilibrium Maxwellian and the distribution function under the ballistic limit, respectively. singular when electron's kinetic energy is small, i.e., when they reside close to the band edge.

To clarify the structure of the singularity involved in Eq. (2), we scale that

$$
\bar{v} \equiv \frac{v}{v_{\text {th }}}, \quad \bar{x} \equiv \frac{x}{v_{\text {th }} \tau_{c}}, \quad \bar{f} \equiv \frac{v_{\text {th }}}{N_{0}} f, \quad \bar{f}_{\text {eq }} \equiv \frac{v_{\text {th }}}{N_{0}} f_{\text {eq }},
$$

where $v_{\mathrm{th}}\left(=\sqrt{k_{B} T_{0} / m}\right)$ is the thermal velocity, $\tau_{c}$ is the relaxation time in the channel region, and $N_{0}$ is the impurity density in high-doped $S$ and $D$ regions. Eliminating the argument $\varepsilon$ for brevity, Eq. (2) reduces to

$$
\bar{v}(\bar{x}) \frac{d \bar{f}(\bar{x})}{d \bar{x}}=-\bar{f}(\bar{x})+\bar{f}_{\mathrm{eq}}(\bar{x}) .
$$

Expanding the electrostatic potential $V(x)$ around $x=x_{b}$, electron's energy $\varepsilon$ is approximated as $\varepsilon \simeq \frac{m v^{2}}{2}-$ $e V\left(x_{b}\right)-\frac{\left(x-x_{b}\right)^{2}}{2} e V^{\prime \prime}\left(x_{b}\right)$. Hence, electron's scaled velocity takes the form

$$
\bar{v}^{2}=\frac{2}{m v_{\mathrm{th}}^{2}}[\varepsilon+e V(x)] \simeq 2\left\{\bar{\delta}+\alpha_{b}\left(\bar{x}-\bar{x}_{b}\right)^{2}\right\},
$$

where $\bar{\delta} \equiv \frac{\varepsilon+e V\left(x_{b}\right)}{k_{B} T_{0}}(\geq 0)$ and $\alpha_{b} \equiv \frac{e \tau_{c}^{2} V^{\prime \prime}\left(x_{b}\right)}{2 m}(>0) . \bar{\delta}$ represents electron's kinetic energy measured from the top of the potential barrier at $x_{b}$ and $\alpha_{b}$ is the parameter. Equation (6) is valid when $\left|\bar{x}-\bar{x}_{b}\right|<\min \left[\Delta \bar{x}_{m}, \bar{x}_{D}-\right.$ $\left.\bar{x}_{b}\right]$ for the left-moving electrons $(v<0)$ or when $\mid \bar{x}-$ $\bar{x}_{b} \mid<\min \left[\Delta \bar{x}_{m}, \bar{x}_{b}-\bar{x}_{S}\right]$ for the right-moving electrons $(v>0)$, where $\Delta \bar{x}_{m} \equiv\left|\frac{3 V^{\prime \prime \prime}\left(x_{b}\right)}{V^{\prime \prime}\left(x_{b}\right)}\right|$.

A rough estimation makes the physical interpretation of the parameter $\alpha_{b}$ clear. Considering the left-moving electrons $(v<0), \alpha_{b}$ is approximately expressed as $\alpha_{b} \sim$ $\frac{e \tau_{c}^{2}\left(V_{a}+\Delta \Phi_{b}\right)}{m L_{c}^{2}} \sim \frac{v_{d} \tau_{c}}{L_{c}}$, where $L_{c}=x_{D}-x_{S}$ and $v_{d}$ is the drift velocity. Therefore, $\alpha_{b}$ characterizes the ballisticity (or diffusivity) of electron transport in the device: the ballistic and diffusive transport correspond to the limit of $\alpha_{b} \rightarrow \infty$ and $\alpha_{b} \rightarrow 0$, respectively. Table I shows typical values of $\alpha_{b}$ for $n^{+}-n-n^{+}$diodes calculated by using the potential profile determined by the conventional driftdiffusion simulations.

Noting that the local equilibrium distribution function is written as $\bar{f}_{\text {eq }}(\bar{x})=\frac{\bar{n}}{\sqrt{2 \pi}} e^{-\left[\bar{\delta}+\alpha_{b}\left(\bar{x}-\bar{x}_{b}\right)^{2}\right]}$ with $\bar{n}=n(x) / N_{0}$, Eq. (5) now takes the form

$$
\left\{\bar{\delta}+\alpha_{b}\left(\bar{x}-\bar{x}_{b}\right)^{2}\right\}^{1 / 2} \frac{d \bar{f}}{d \bar{x}}=\frac{1}{\sqrt{2}}\left[\bar{f}-\frac{\bar{n}}{\sqrt{2 \pi}} e^{-\left\{\bar{\delta}+\alpha_{b}\left(\bar{x}-\bar{x}_{b}\right)^{2}\right\}}\right]
$$

for the left-moving electrons $(v<0)$. Hereafter, we only consider the case $v<0\left(\bar{x}>\bar{x}_{b}\right)$, which is responsible for generating the negative-velocity component of the distribution function $f\left(x_{b}, v<0\right)$. We would like to stress again that the coefficient of the first derivative in Eq. (7) could be indefinitely small when the kinetic en- 
ergy $\bar{\delta}$ is small and electron approaches the top of the potential barrier at $\bar{x}_{b}$. It is well known that the solution for such differential equations exhibits the boundary layer structure in which the solution changes very rapidly in the layer region near $x_{b}$ [8]. It is, therefore, crucial to keep in mind the magnitude of both $\bar{\delta}$ and $\alpha_{b}\left(\bar{x}-\bar{x}_{b}\right)^{2}$ when it is expanded.

We now derive an analytical solution for Eq. (7). We suppose that $\alpha_{b}$ is large (quasiballistic regime). We write the coefficient of the first derivative in Eq. (7) as

$$
\begin{aligned}
\left\{\bar{\delta}+\alpha_{b}\left(\bar{x}-\bar{x}_{b}\right)^{2}\right\}^{1 / 2} \approx & \alpha_{b}^{1 / 2}\left(\bar{x}-\bar{x}_{b}\right) \\
& \times\left\{1+\frac{\bar{\delta}}{2 \alpha_{b}\left(\bar{x}-\bar{x}_{b}\right)^{2}}+\ldots\right\} .
\end{aligned}
$$

Notice that the expansion is nonuniform for $\left|\bar{x}-\bar{x}_{b}\right|=$ $O\left(\sqrt{\bar{\delta} / \alpha_{b}}\right)$ and thus, valid only when $\left|\bar{x}-\bar{x}_{b}\right|>\Delta \bar{x}_{0}$ with $\Delta \bar{x}_{0} \equiv \sqrt{\bar{\delta} / \alpha_{b}}$. In the allowed region such that $\left|\bar{x}-\bar{x}_{b}\right|>$ $\Delta \bar{x}_{0}$ (Region I), Eq. (7) has the following form

$$
\alpha_{b}^{\frac{1}{2}}\left(\bar{x}-\bar{x}_{b}\right) \frac{d \bar{f}}{d \bar{x}}=\frac{1}{\sqrt{2}}\left\{\bar{f}-\frac{\bar{n}}{\sqrt{2 \pi}} e^{-\alpha_{b}\left(\bar{x}-\bar{x}_{b}\right)^{2}}\right\} .
$$

Assuming that $\bar{n}$ is constant in the channel region, the solution for Eq. (9) is given by

$$
\begin{aligned}
\bar{f}_{I}(\bar{x})= & \frac{\bar{n}}{4 \sqrt{\pi \alpha_{b}}}\left\{\left\{\alpha_{b}\left(\bar{x}-\bar{x}_{b}\right)^{2}\right\}^{1 / \sqrt{8 \alpha_{b}}}\right. \\
& \times \Gamma\left[-\frac{1}{\sqrt{8 \alpha_{b}}}, \alpha_{b}\left(\bar{x}-\bar{x}_{b}\right)^{2}\right] \\
& -\left(\frac{\bar{x}-\bar{x}_{b}}{\bar{x}_{m}-\bar{x}_{b}}\right)^{1 / \sqrt{8 \alpha_{b}}}\left[\alpha_{b}\left(\bar{x}_{m}-\bar{x}_{b}\right)^{2}\right]^{1 / \sqrt{8 \alpha_{b}}} \\
& \left.\times \Gamma\left[-\frac{1}{\sqrt{8 \alpha_{b}}}, \alpha_{b}\left(\bar{x}_{m}-\bar{x}_{b}\right)^{2}\right]\right\},
\end{aligned}
$$

where $\Gamma(a, z)$ is the incomplete gamma function defined by $\Gamma(a, z)=\int_{z}^{\infty} d t e^{-t} t^{a-1}$. To obtain the solution, we have assumed that the distribution function $\bar{f}_{I}(\bar{x})$ decays to zero as $\bar{x}$ approaches $\bar{x}_{m}$, where $\bar{x}_{m}=\min \left(\bar{x}_{b}+\right.$ $\left.\Delta \bar{x}_{m}, \bar{x}_{D}\right)$. This is justified because the number of ballistic electrons reaching the potential barrier at $x_{b}$ from the $D$ region is negligibly small if the applied voltage $V_{a}$ is much greater than $k_{B} T_{0} / e$.

In the other region such that $\left|\bar{x}-\bar{x}_{b}\right|<\Delta \bar{x}_{0}$ (Region II), $\bar{\delta}>\alpha_{b}\left(\bar{x}-\bar{x}_{b}\right)^{2}$ and thus we may approximate Eq. (7) as

$$
\bar{\delta}^{1 / 2} \frac{d \bar{f}}{d \bar{x}}=\frac{1}{\sqrt{2}}\left\{\bar{f}-\frac{\bar{n}}{\sqrt{2 \pi}} e^{-\bar{\delta}}\right\} .
$$

The solution for Eq. (11) is given by

$$
\bar{f}_{I I}(\bar{x})=\frac{\bar{n}}{\sqrt{2 \pi}} e^{-\bar{\delta}}\left(1-e^{-\bar{x}_{0}-\bar{x} / \sqrt{2 \delta}}\right)+\bar{f}_{I}\left(\bar{x}_{0}\right) e^{-\bar{x}_{0}-\bar{x} / \sqrt{2 \delta}},
$$

TABLE I. Ballisticity parameter $\alpha_{b}$ and the size of the transition region $\Delta \bar{x}_{0}$ for $n^{+}-n-n^{+}$diodes. It is assumed that the kinetic energy at $x=x_{b}$ is $\bar{\delta}=1$, the applied voltage is $V_{a}=0.2 \mathrm{~V}$, the effective mass is $m=0.322 m_{e}\left(m_{e}\right.$ : electron mass), the relaxation time is $\tau_{c}=50 \mathrm{fs}$, and the doping densities in $n^{+}$and $n$ regions are $10^{20}$ and $10^{18} \mathrm{~cm}^{-3}$, respectively.

\begin{tabular}{lll}
\hline \hline$L_{c}(\mathrm{~nm})$ & $\alpha_{b}$ & $\Delta \bar{x}_{0}\left(\Delta x_{0} \mathrm{~nm}\right)$ \\
\hline 10 & 5.978 & $0.4090(2.429)$ \\
15 & 3.651 & $0.5233(3.109)$ \\
30 & 1.406 & $0.8434(5.010)$ \\
50 & 0.7210 & $1.178(6.996)$ \\
100 & 0.3459 & $1.700(10.10)$ \\
200 & 0.1694 & $2.430(14.43)$ \\
\hline \hline
\end{tabular}

where $\bar{x}_{0}=\bar{x}_{b}+\Delta \bar{x}_{0}$ and we have assumed that $\lim _{\bar{x} \rightarrow \bar{x}_{0}-0} \bar{f}_{I I}(\bar{x})=\lim _{\bar{x} \rightarrow \bar{x}_{0}+0} \bar{f}_{I}(\bar{x})$. Using the Heaviside unit step function $\theta(x)$, the velocity distribution function $\bar{f}(\bar{x})$ for $\bar{x} \geq \bar{x}_{b}$ is finally given by

$$
\bar{f}(\bar{x})=\bar{f}_{I}(\bar{x}) \theta\left(\bar{x}-\bar{x}_{0}\right)+\bar{f}_{I I}(\bar{x}) \theta\left(\bar{x}_{0}-\bar{x}\right) .
$$

From the above derivation of the analytical solution of the BTE, it is clear that the size of the transition region $\Delta \bar{x}_{0}$, which corresponds to the size of Region II, plays a crucial role in determining the velocity distribution function $f(x, v<0)$. The size of $\Delta \bar{x}_{0}$ for $n^{+}-n-n^{+}$diodes with various $L_{c}$ is listed in Table I. When $L_{c}$ is large so that $\alpha_{b}<1$ (diffusive regime), $\Delta \bar{x}_{0}$ is large and Region II dominates in shaping the velocity distribution function $f\left(x_{b}, v<0\right)$. This implies that the electrons around the potential barrier are thermalized and $f\left(x_{b}, v<0\right)$ is approximated by the thermal equilibrium distribution. On the other hand, as $L_{c}$ shrinks, $\Delta \bar{x}_{0}$ becomes small. However, Region II cannot be ignored because it is also associated with the layer region.

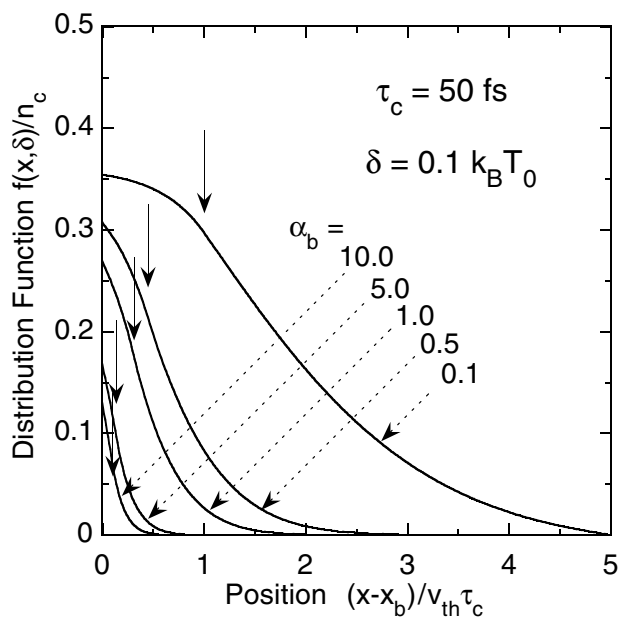

FIG. 3. Distribution function $f(x, v<0)$ with fixed $\bar{\delta}=0.1$ along the channel for $\alpha_{b}=0.1,0.5,1,5,10$. The vertical arrows represent the boundary between Region I and II. 


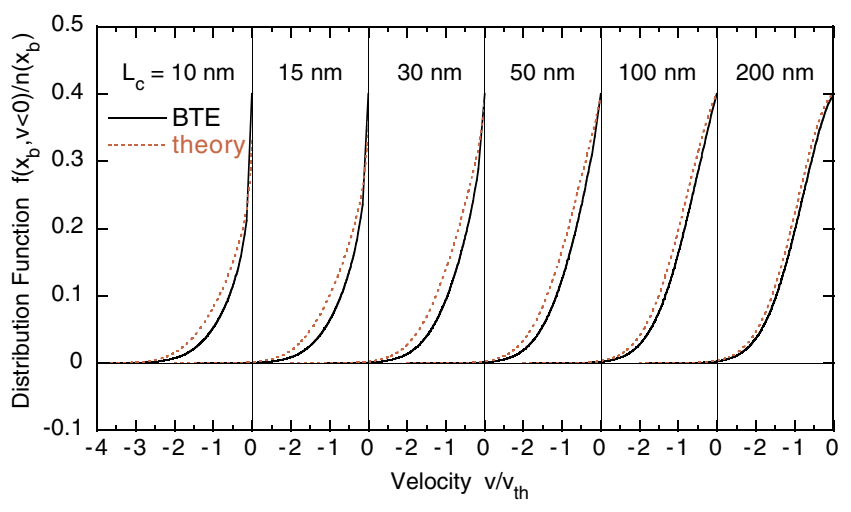

FIG. 4 (color online). Velocity distribution functions $f\left(x_{b}, v<0\right)$ obtained from the present theory (dotted lines) and from the BTE (solid lines). The values of $\alpha_{b}$ listed in Table I have been used.

Figure 3 shows how the distribution function with negative-component velocity $f(x, v<0)$ grows as electrons approach the potential barrier at $x=x_{b}$ from the right $D$ region for various $\alpha_{b}$. The kinetic energy at $x=$ $x_{b}$ is fixed to $\bar{\delta}=0.1$. The solution grows rapidly near the top of the potential barrier at $x_{b}$ and indeed exhibits a boundary layer structure. Even when $\alpha_{b}$ is large, $f(x, v<$ $0)$ grows very rapidly in a thin region, whose length is much smaller than the mean-free path $(\approx 6 \mathrm{~nm})$. This tendency is particularly strong as $\bar{\delta}$ is small, i.e., as electrons reside close to the band edge at $x=x_{b}$. Therefore, Region II as well as Region I cannot be ignored no matter how small $L_{c}$ is. Notice that the number of electrons coming ballistically from the $D$ region is negligible and thus the ballistic limit corresponds to ignoring both Region I and II.

Figure 4 shows the velocity distribution functions at the top of the potential barrier $f\left(x_{b}, v<0\right)$ obtained from Eq. (13) and from the numerical calculations of the BTE for $n^{+}-n-n^{+}$diodes with various channel lengths $L_{c}$. Note that the present theory contains essentially no adjustable parameter. The agreement between the two results is excellent and it justifies the assumption that $\bar{f}(\bar{x})$ decays to zero as $\bar{x}$ approaches $\bar{x}_{m}$. In other words, $f\left(x_{b}, v<0\right)$ entirely results from scatterings in the channel region. This is physically interpreted as follows. When electrons pass around the top of the potential barrier, their velocities could be indefinitely small. As a result, electrons spend longer time around the potential barrier and suffer scatterings. These electrons are, therefore, quasithermal and have the same origin found in longer devices.

The present analyses entirely rely on the RTA. Since the key idea lies in the singular nature inherent in the BTE under the RTA, the question is whether a singularity remains present in the BTE with the full collision integral. Considering the fact that the full collision integral could be reduced to the RTA if the band structure is isotropic and the scattering is elastic [9], we expect that a similar singularity is still preserved in the full BTE. However, we should mention that, if elastic acoustic phonon scattering is the only dominant scattering, the scattering rate approaches to zero as electron energy diminishes. In this case, the present arguments would break down and the energy-dependent RTA should be employed [10]. Nevertheless, in nonpolar semiconductors such as bulk Si or Ge, optical phonon scattering (absorption) among the equivalent valleys also takes place at room temperature. In polar semiconductors, the polar optical phonon scattering (absorption) is active. The scattering time is then finite in both cases even when electron energy becomes close to zero. Therefore, we expect that the present results would not be changed significantly in many cases unless inelastic scatterings are prohibited somehow [11].

In conclusion, we have theoretically investigated quasiballistic electron transport in nanoscale semiconductor structures by solving the BTE under the RTA. It has been shown that the solution of the BTE exhibits a boundary layer structure near the potential barrier and the scatterings in the channel region cannot be neglected even in nanoscale structures.

*Electronic address: sano@esys.tsukuba.ac.jp

[1] F. G. Pikus and K. K. Likharev, Appl. Phys. Lett. 71, 3661 (1997).

[2] M. Lundstrom, IEEE Electron Device Lett. 18, 361 (1997); IEEE Electron Devices 49, 133 (2002).

[3] G. Gomila, I. R. Cantalapiedra, T. González, and L. Reggiani, Phys. Rev. B 66, 075302 (2002).

[4] M. Naspreda, O. M. Bulashenko, and J. M. Rubi, Phys. Rev. B 68, 155321 (2003).

[5] P. Resiboir and M. de Leener, Classical Kinetic Theory of Fluids (John Wiley, New York, 1977).

[6] N. Sano, Appl. Phys. Lett. 85, 4208 (2004).

[7] H. U. Baranger and J.W. Wilkins, Phys. Rev. B 30, R7349 (1984).

[8] A. H. Nayfeh, Perturbation Methods (John Wiley, New York, 1973).

[9] N.W. Aschcroft and N. D. Mermin, Solid State Physics (Saunders College, Philadelphia, 1976), p. 324.

[10] If elastic acoustic phonon scattering is the only dominant scattering, the scattering rate decreases as $\bar{\delta}^{1 / 2}$ so that $v \tau_{c}(\bar{\delta})$ is finite at $\bar{\delta}=0$. Then, the boundary layer structure would cease to exist.

[11] Another issue which should be addressed is on the use of semiclassical kinetic equation. The length scale concerned is small and quantum mechanical treatments should be employed. However, if inelastic phonon scattering is effective at room temperature, the phase coherence of electron waves propagating in the channel is disturbed around the potential barrier, which may validate the semiclassical treatment. However, we would like to stress that further studies based on quantum kinetic theory are needed to draw any decisive conclusions. 